\title{
Modeling studies of heterogeneities in glycolytic oscillations in HeLa cervical cancer cells
}

Cite as: Chaos 29, 033132 (2019); https://doi.org/10.1063/1.5087216

Submitted: 29 December 2018 . Accepted: 08 March 2019 . Published Online: 28 March 2019

Takashi Amemiya (D), Kenichi Shibata (D), Yichen Du, Satoshi Nakata, and Tomohiko Yamaguchi

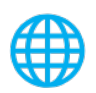

\section{Don't let your writing} keep you from getting published!

AIP Author Services 


\title{
Modeling studies of heterogeneities in glycolytic oscillations in HeLa cervical cancer cells
}

\author{
Cite as: Chaos 29, 033132 (2019); doi: 10.1063/1.5087216 \\ Submitted: 29 December 2018 . Accepted: 8 March 2019. \\ Published Online: 28 March 2019

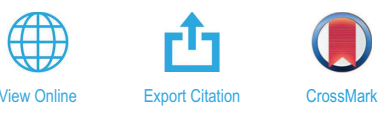 \\ Takashi Amemiya, ${ }^{1, a)}$ (D) Kenichi Shibata, ${ }^{1}$ (D) Yichen Du, ${ }^{7}$ Satoshi Nakata, ${ }^{2}$ and Tomohiko Yamaguchi ${ }^{3}$

\begin{abstract}
AFFILIATIONS
${ }^{1}$ Graduate School of Environment and Information Sciences, Yokohama National University (YNU), 79-7 Tokiwadai, Hodogaya-ku, Yokohama, Kanagawa 240-8501, Japan

${ }^{2}$ Graduate School of Science, Hiroshima University, 1-3-1 Kagamiyama, Higashihiroshima 739-8526, Japan
\end{abstract} \\ ${ }^{3}$ Meiji Institute for Advanced Study of Mathematical Sciences (MIMS), 4-21-1 Nakano, Nakano-ku, Tokyo 164-8525, Japan
}

Note: This paper is part of the Focus Issue, "Nonlinear Chemical Dynamics and Its Interdisciplinary Impact: Dedicated to Ken Showalter on the Occasion of his 70th Birthday."

a) Author to whom correspondence should be addressed: amemiya-takashi-jk@ynu.ac.jp

\begin{abstract}
Previous experiments demonstrated that a population of HeLa cells starved of glucose or both glucose and serum exhibited a strong heterogeneity in the glycolytic oscillations in terms of the number of oscillatory cells, periods of oscillations, and duration of oscillations. Here, we report numerical simulations of this heterogeneous oscillatory behavior in HeLa cells by using a newly developed mathematical model. It is simple enough that we can apply a mathematical analysis, but capture the core of the glycolytic pathway and the activity of the glucose transporter (GLUT). Lognormal distributions of the values of the four rate constants in the model were obtained from the experimental distributions in the periods of oscillations. Thus, the heterogeneity in the periods of oscillations can be attributed to the difference in the rate constants of the enzymatic reactions. The activity of GLUT is found to determine whether the HeLa cells were oscillatory or non-oscillatory under the same experimental conditions. Simulation with the log-normal distribution of the maximum uptake velocity of glucose and the four randomized rate constants based on the log-normal distributions successfully reproduced the time-dependent number of oscillatory cells (oscillatory ratios) under the two starving conditions. The difference in the initial values of the metabolites has little effect on the simulated results.
\end{abstract}

Published under license by AIP Publishing. https://doi.org/10.1063/1.5087216

In biochemical reactions, oscillations in the concentrations of metabolites have been observed during glycolysis, and this phenomenon is known as glycolytic oscillations. They have been observed in cardiac, pancreatic, muscle, yeast, and cancer cells. Some of the oscillations are also known to synchronize between cells via intercellular interaction. Two routes to synchronization have been identified in a population of yeast cells: one is the dynamical quorum-sensing transition from the quiescent to the oscillatory states, and the other is known as the Kuramoto transition from desynchronized to synchronized oscillations. To date, the former has been observed only in well-stirred suspensions of yeast cells, whereas the latter has been observed only in immobilized yeast cells on a coverslip. Showalter and co-workers have revealed that both types of transitions occur in large populations of chemical oscillators, suggesting that suspensions of cellular organisms, such as yeast or Dictyostelium discoideum, may also undergo these two types of transitions. Here, we show modeling studies of glycolytic oscillations in HeLa cervical cancer cells grown in a monolayer on a slide chamber, whose experiments were individually carried out earlier. Previous experiments demonstrated that individual HeLa cells exhibited heterogeneous glycolytic oscillations with different periods of oscillations, and thus the collective behavior was asynchronous with very low values of the order parameter. The present study presents a new mathematical model for the glycolytic oscillations in HeLa cells, and the numerical simulations and their analysis reveal the existence of heterogeneity in the enzymatic reactions in the glycolytic pathway.

\section{INTRODUCTION}

Glycolysis is a primordial process to acquire energy by producing adenosine triphosphate (ATP) in a living cell. Glycolytic oscillations, in which the concentrations of metabolites of the glycolytic pathway oscillate, may occur under certain conditions and have been 
the focus for theoretical and experimental studies in rhythmic behaviors on the cellular level (Goldbeter, 1996). Glycolytic oscillations have been studied intensively by using yeast, Saccharomyces cerevisiae, as a model organism of eukaryotes in order to elucidate the intracellular oscillations and the intercellular synchronization (Danø et al., 1999; De Monte et al., 2007; Olsen et al., 2009; Gustavsson et al., 2012; Weber et al., 2012; Amemiya et al., 2015; and Shibata et al., 2018). Two routes to synchronization, dynamical quorum sensing (De Monte et al., 2007) and Kuramoto desynchronization (Shinomoto and Kuramoto, 1986 and Weber et al., 2012), have been revealed in glycolytic oscillations in yeast cells, as well as oscillations in large populations of chemical oscillators (Taylor et al., 2009).

Yeasts are also known to enhance their glycolytic pathway even under aerobic conditions and high external glucose concentrations by impairing oxidative phosphorylation; this is known as the Crabtree effect (Crabtree, 1929). On the other hand, many types of cancer cells are also known to enhance their glycolytic activity under aerobic conditions; this is known as the Warburg effect (Warburg, 1956 and Diaz-Ruiz et al., 2011). Since the Warburg effect is a metabolic hallmark of cancer cells (Heiden et al., 2009), glycolysis has been the focus of research interest, even from a therapeutic viewpoint of cancer (Hamanaka and Chandel, 2012).

We have focused on the metabolic similarity between yeast and cancer cells and carried out experiments of glycolytic oscillations in HeLa cervical cancer cells as a model system. As expected, we could observe the oscillations in the individual HeLa cells for the first time (Amemiya et al., 2017). We have found the following three characteristic behaviors in HeLa glycolytic oscillations: (i) starvation of glucose (pre-incubation with no glucose) was indispensable for the oscillations, which is the same as for yeast glycolytic oscillations; (ii) starvation of both glucose and serum induced a strong heterogeneity in their oscillations with longer periods and larger amplitudes than those under only glucose starvation; and (iii) quantitative analysis of the intercellular synchronization by evaluating the Kuramoto order parameter $K(t)$ (Shinomoto and Kuramoto, 1986) revealed that the HeLa cells exhibited asynchronous collective behaviors with very low $K(t)$ values, indicating that the coupling between the cells was very weak under these conditions.

The heterogeneity and asynchronicity observed in HeLa cells may be the cause why there are no reports of glycolytic oscillations in cancer cells, except for only a preliminary note (in distinct glycolytic oscillations in Ehrlich ascites tumor; Ibsen and Schiller, 1967); even though glycolysis in cancer cells has extensively been studied using aerobic suspensions of glucose-starved cells (Wu and Racker, 1959 and Chance and Hess, 1959). Namely, if the asynchronization in glycolytic oscillations observed in HeLa cells is typical in cancer cells, this makes it difficult to explore the glycolytic oscillations in systems of cell suspensions, because no collective oscillations can be observed in those systems. Contrarily, a system of monolayered cells, which was previously developed in the studies of yeast (Weber et al., 2012 and Gustavsson et al., 2012), enabled us to observe oscillations in individual HeLa cells even though their oscillations were asynchronous.

To date, the mechanisms of the glycolytic oscillations in yeasts have been elucidated based on the enzymatic reactions within the cells (Goldbeter, 1996 and Richard et al., 1996). Many mathematical models have been developed to reproduce the glycolytic oscillations, from minimal models with two variables (Sel'kov, 1968; Goldbeter and Lefever, 1972; Schütze and Wolf, 2010; and Chandra et al., 2011) to models with five to nine variables (Termonia and Ross, 1981; Wolf and Heinrich, 2000; and Wolf et al., 2000), and models with extensive mechanistic details (du Preez et al., 2012 and Hald and Sørensen, 2010). Additionally, very detailed models for cancer glycolysis have been developed to investigate the basic cellular physiology such as enzymatic and transport properties (Marín-Hernández et al., 2011; Khazaei et al., 2012; and Marín-Hernández et al., 2014); however, no glycolytic oscillations have been investigated in these models. A kinetic model was proposed recently to reproduce glycolytic oscillations of HeLa cells qualitatively (Martin et al., 2017).

The present study aims to use a simple model developed here to describe the heterogeneity in glycolytic oscillations in HeLa cells observed in our previous study (Amemiya et al., 2017). The present model can identify biochemically specific mechanisms for glycolytic oscillations and quantitatively reproduce the heterogeneous oscillatory behaviors by taking into account the variation in the rate constants for the enzymatic reactions and in the flux of glucose uptake through the glucose transporter (GLUT).

\section{MATHEMATICAL MODEL}

\section{Model scheme}

We have developed a simple model in order to simulate the characteristic heterogeneous glycolytic oscillations in HeLa cells as shown in Fig. 1. The present model captures the core of the glycolytic pathway and is simple enough that we can apply a mathematical analysis. Namely, it takes into account the upstream (ATP-consuming) reactions of hexose and the downstream (ATP-producing) reactions of triose (Chandra et al., 2011). Allosteric reactions of phosphofructokinase $(\mathrm{PFK})$ and pyruvate kinase $(\mathrm{PK})$ represent the upstream (ATP-consuming) and downstream (ATP-producing) processes, respectively. Several glycolytic reactions are lumped, and the model variables denote the concentrations of pools of intermediates, rather than the concentrations of individual compounds for simplicity. The present model describes Michaelis-Menten type mechanisms for the PFK and PK reactions (Sel'kov, 1968 and Termonia and Ross, 1981) and a first-order reaction for the consumption of ATP and the final product (Wolf et al., 2000).

The present model has also included the uptake of glucose into the cells through the glucose transporter (GLUT). Glucose uptake is reported to enhance glycolysis in more than 20-fold in HeLa cells starved under the no-glucose condition for $24 \mathrm{~h}$ (Shaw and Amos, 1973). HeLa cells were also starved without glucose for $24 \mathrm{~h}$ in our previous study (Amemiya et al., 2017), and glucose-starvation was indispensable for glycolytic oscillations. Thus, this effect was modeled by changing the flux of glucose-uptake through GLUT.

Another characteristics in the present model is that the concentration of glucose in the extracellular solution, $G_{\mathrm{ex}}$, was taken into account. This variable is indispensable for reproducing the in vitro experiments performed in our previous study and yeast glycolytic oscillations (Weber et al., 2012; Amemiya et al., 2015; Dodd and Kralj, 2017; and Shibata et al., 2018). In such experiments, glucose is introduced, at a certain time for few seconds, into a chamber or a vessel in which glucose-starved cells are immobilized or suspended. Afterwards, the cells start to take up the extracellular glucose 


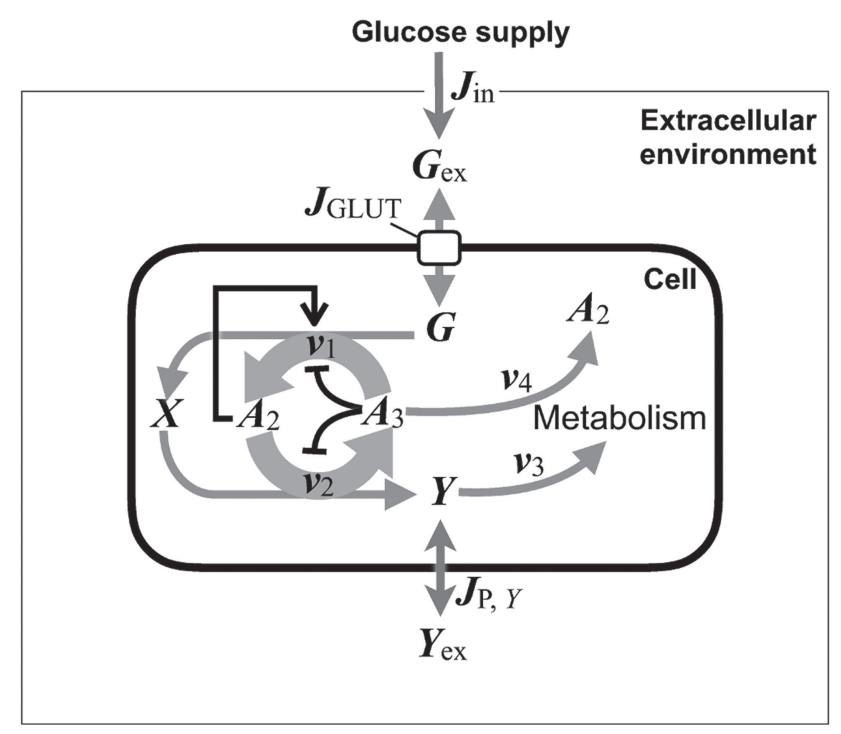

FIG. 1. Schematic representation of the model. Variables are $G_{\text {ex }}$ (extracellular glucose), $G$ (glucose), $X$ (pools of intermediates after the PFK reaction), $Y$ (pools of intermediates after the PK reaction), $Y_{\text {ex }}$ (extracellular $Y$ ), and $A_{3}$ (ATP). Total concentration of $A_{2}(\mathrm{ADP})$ and $A_{3}$ is assumed to be constant: $A_{2}+A_{3}=A_{0}$. Transport processes are $J_{\text {in }}$ (glucose supply), $J_{G L U T}$ (glucose transporter), and $J_{P, Y}$ (exchange of $Y$ across the plasma membrane). Reaction rates of enzymatic reactions are $v_{1}$ (PFK reaction), $v_{2}$ (PK reaction), $v_{3}$ (consumption of $Y$ ), and $v_{4}$ (ATP consumption). The PKF reaction $\left(v_{1}\right)$ is activated by $A_{2}$ and inhibited by $A_{3}$. The PK reaction $\left(v_{2}\right)$ is inhibited by $A_{3}$. PFK: phosphofructokinase, PK: pyruvate kinase.

through GLUT. The present model can reproduce such experimental conditions and the cellular processes that affect oscillatory dynamics.

\section{Model kinetics}

We propose a model with four chemical reactions and three transport processes (including one injection), as shown in Fig. 1: flux $J_{\text {in }}$, external input of glucose to the extracellular solution; flux $J_{\mathrm{GLUT}}$, transport of the extracellular glucose into the cell through the glucose transporter GLUT; reaction 1 (the reaction rate is denoted by $v_{1}$ ), the PFK reaction that represents up-stream (ATP-consuming) reactions in glycolysis; reaction $2\left(v_{2}\right)$, the PK reaction that represents down-stream (ATP-producing) reactions in glycolysis; reaction $3\left(v_{3}\right)$, consumption reaction of the final product; reaction $4\left(v_{4}\right)$, non-glycolytic ATP consumption; flux $J_{\mathrm{P}, Y}$, membrane transport of triose including lactate that is the final product of fermentation.

First, we show the three-transport kinetics. Glucose influx into the extracellular solution was denoted by $J_{\text {in }}$. Under experimental conditions similar to the present study, a glucose solution is added into a batch chamber in a pulse-like manner from time $t_{1}$ to $t_{2}$ so that the concentration of glucose be $G_{\mathrm{i}}(\mathrm{mM})$, thus $J_{\text {in }}$ is represented by

$$
J_{\text {in }}= \begin{cases}\frac{G_{i}}{t_{2}-t_{1}}, & t_{1} \leq t \leq t_{2} \\ 0 & \text { otherwise }\end{cases}
$$

It is noted that in the case of continuous flow from an outer source, with a concentration of $G_{s}(\mathrm{mM}), J_{\text {in }}$ can be replaced by

$$
J_{\text {in }}=\gamma\left(G_{\mathrm{s}}-G_{\text {ex }}\right) \text {, }
$$

where $\gamma$ is a constant. The present study uses Eq. (1a) instead of Eq. (1b) according to the previous experimental conditions.

The kinetics of GLUT was introduced as a mono-substrate reversible Michaelis-Menten equation (Marín-Hernández et al., 2011)

$$
J_{\mathrm{GLUT}}=V_{\mathrm{max}} \frac{G_{\mathrm{ex}}-\frac{G}{K_{\mathrm{eq}}}}{K_{\mathrm{out}}\left(1+\frac{G}{K_{\mathrm{in}}}\right)+G_{\mathrm{ex}}},
$$

where $K_{\text {out }}$ and $K_{\text {in }}$ are the affinity constants of the enzyme $\left(K_{\mathrm{m}}\right.$ values in the Michaelis-Menten mechanism) for extra- and intracellular glucose, respectively; $K_{\mathrm{eq}}$ is the equilibrium constant; $V_{\max }$ is the maximum velocity in the forward reaction that may increase by glucose-starvation (Shaw and Amos, 1973).

The flux of the final product, $Y$, across the plasma membrane is considered to be proportional to the difference between intra- and extra-cellular concentrations

$$
J_{\mathrm{P}, Y}=\kappa\left(Y-Y_{\mathrm{ex}}\right),
$$

where $\kappa$ is a coupling constant and is represented by $\kappa=S P / V$, in which $S$ is the cell surface area, $P$ is the permeability of the membrane, and $V$ is the cellular volume (Wolf and Heinrich, 2000).

Second, we show the enzymatic reactions. We assumed a Michaelis-Menten type mechanism for the enzymatic reactions of PFK and PK. For the PFK reaction, the enzymatic processes including the inhibition by its substrate ATP and the activation by its product $\mathrm{ADP}$ are considered, and the reaction rate $\left(v_{1}\right)$ is expressed by a function (see the Appendix)

$$
f\left(G, A_{3}\right)=\frac{A_{2}{ }^{m}}{\left[1+A_{2}{ }^{m}\left(\frac{1}{K_{1}}+\frac{G}{K_{1} K_{3}}+\frac{A_{3}}{K_{1} K_{4}}\right)+\frac{A_{3}{ }^{m}}{K_{2}}\right]},
$$

where $A_{2}$ and $A_{3}$ represent [ADP] and [ATP], respectively, and $A_{2}+A_{3}=A_{0}$ was assumed to be constant; $K_{1}$ and $K_{2}$ are dissociation constants of PFK-ATP and PFK-ADP complexes, respectively; $m$ is the number of PFK subunits; $K_{3}$ and $K_{4}$ are also dissociation constants for the subsequent enzyme-substrate complexes. On the other hand, for the PK reaction, only inhibition by its product ATP is considered, and the reaction rate $\left(v_{2}\right)$ is expressed with another function

$$
g\left(X, A_{3}\right)=\frac{1}{\left(1+\frac{A_{3}^{n}}{K_{5}}+\frac{X}{K_{6}}+\frac{A_{2}}{K_{7}}\right)},
$$

where $K_{5}$ is a dissociation constant of the PK-ATP complex; $n$ is the number of PK subunit; $K_{6}$ and $K_{7}$ are also dissociation constants of the subsequent enzyme-substrate complexes.

Thus, the four reaction rates read as

$$
\begin{aligned}
& v_{1}=k_{1} G A_{3} f\left(G, A_{3}\right), \\
& v_{2}=k_{2} X A_{2} g\left(X, A_{3}\right), \\
& v_{3}=k_{3} Y, \\
& v_{4}=k_{4} A_{3} .
\end{aligned}
$$


Assuming that the metabolites are distributed homogeneously in the cell and in the extracellular solution, the system of the differential equations of the model reads as

$$
\begin{aligned}
& \frac{\mathrm{d} G}{\mathrm{~d} t}=J_{\mathrm{GLUT}}-v_{1}, \\
& \frac{\mathrm{d} X}{\mathrm{~d} t}=v_{1}-v_{2}, \\
& \frac{\mathrm{d} Y}{\mathrm{~d} t}=2 v_{2}-v_{3}-J_{\mathrm{P}, Y}, \\
& \frac{\mathrm{d} A_{3}}{\mathrm{~d} t}=-2 v_{1}+4 v_{2}-v_{4}, \\
& \frac{\mathrm{d} G_{\mathrm{ex}}}{\mathrm{d} t}=J_{\mathrm{in}}-\varphi J_{\mathrm{GLUT}}, \\
& \frac{\mathrm{d} Y_{\mathrm{ex}}}{\mathrm{d} t}=\varphi J_{\mathrm{P}, Y},
\end{aligned}
$$

where $\varphi$ denotes the ratio of the cellular volume $(V)$ to the extracellular volume $\left(V_{\text {ex }}\right)$ (Wolf and Heinrich, 2000). Factors 2 and 4, which multiply $v_{2}$ in Eqs. (7c) and (7d), respectively, come from the fact that two molecules of $Y$ (pool of triose) and four molecules of ATP are produced each time one molecule of $X$ is converted into the product of $Y$ in the downstream glycolysis; and factor -2 , which multiplies $v_{1}$ in Eq. (7d), comes from the fact that two molecules of ATP are consumed each time one molecule of glucose $(G)$ is converted into $X$ in the upstream glycolysis.

\section{RESULTS}

\section{Simulation of individual oscillations}

We simulated individual glycolytic oscillations in HeLa cells pre-incubated with (i) (Glc-, FBS+), (ii) (Glc-, FBS-), (iii) (Glc+, FBS+) (control), and (iv) (Glc+, FBS-), where Glc- and Glc + indicate without and with glucose, respectively; and FBS- and FBS+ indicate without and with serum, respectively. The results are shown in Fig. 2. Briefly, the characteristic behaviors under the four conditions were (i) the periods of oscillations were shorter under (Glc-, FBS+), (ii) the periods were longer under (Glc-, FBS-), and (iii) no oscillations were exhibited after pre-incubation with glucose.

We set the values of the parameters as shown in the caption in Fig. 2 as follows: the values of $A_{0}, k_{i}, V_{\max }, K_{\text {eq }}, K_{\text {out }}, K_{\text {in }}, \kappa, \varphi$, $m$, and $n$ were assigned by referring to the literature (Wolf et al., 2000; Marín-Hernández et al., 2014; and Shaw and Amos, 1973), whereas the dissociation constants $K_{1}-K_{7}$ were newly assigned in the present study with reference to the literature (Marín-Hernández et al., 2011; 2014). The present model can reproduce experimental behaviors quantitatively with the parameter set. First, the effect of serum-starvation (Glc-, FBS-) was reproduced by using smaller values of the rate constants $\left(k_{1}-k_{4}\right)$ than those under only glucosestarving condition (Glc-, FBS+), yielding oscillations with larger periods as shown in panel (b) than those in panel (a). The effect of FBS- on the values of the rate constants was assumed from a study reporting that the enzymatic activity of PFK was increased by the serum addition to serum-starved cultures of human fibroblasts (Bruni et al., 1983). It is noted that the same activity of GLUT was used under the two conditions.
Second, the effect of no-starvation of glucose, (Glc+, FBS+) and (Glc+, FBS-), was reproduced by using a small value of $V_{\max }$, i.e., decrease in the activity of GLUT, exhibiting very small spikelike response upon addition of glucose and no subsequent oscillation [panels (c) and (d)]. The effect of serum-starvation (FBS-) affected little under the two conditions. These results confirm that glucosestarvation (Glc-) induces high uptake of glucose through GLUT (Shaw and Amos, 1973), which must be indispensable for the glycolytic oscillations in HeLa cells. Third, oscillations with different periods were obtained by using different values of the rate constants, and oscillations with different starting time and duration of the oscillations were reproduced by using different values of the rate constants and different initial concentrations of the metabolites in the cells.

\section{Distribution of the periods of the oscillations}

Previous study (Amemiya et al., 2017) showed that the distribution in the periods of oscillations was different depending on the two starving conditions: (Glc-, FBS+) and (Glc-, FBS-). HeLa cells starved with both glucose and serum (Glc-, FBS-) exhibited longer mean period and broader period distribution than cells starved with only glucose (Glc-, FBS+). Period distributions originate from the difference in the enzymatic activity in the glycolytic pathway in individual cells.

Here, we try to evaluate the distribution in the values of the rate constants $\left(k_{1}-k_{4}\right)$ in the present model (Fig. 1) from the experimentally obtained distributions in the periods of the oscillations. Thus, we first defined the minimum and maximum values of each of the four rate constants as listed in Table I, which can generate oscillations similar to the experimental ones. Second, a new parameter $\alpha$ was introduced to yield the values of the four rate constants as

$$
k_{\mathrm{i}}=a_{\mathrm{i}} \alpha+b_{\mathrm{i}}, \quad \mathrm{i}=1,2,3,4,
$$

where $a_{\mathrm{i}}$ and $b_{\mathrm{i}}$ are constants as listed in Table I. Equation (8) is introduced in such a way that each $k_{\mathrm{i}}$ can take values from its minimum to maximum values when $\alpha$ is changed from 0.05 to 1 , respectively. Equation (8) assumes that the values of the four rate constants can be different among the HeLa cells; however, they are not totally random; namely, it excludes the case that the activity of one of the enzymes was very high, whereas the activity of one of the other three enzymes was very low in a cell. This assumption was made with reference to a few studies that the activities of some glycolytic enzymes are often regulated synchronously; In human colorectal carcinoma LoVo cells, glycolytic enzymes such as hexokinase (HK), phosphofructokinase (PFK), and pyruvate kinase (PK) are down regulated at the transcriptional level under serum starvation (Zheng et al., 2016). Furthermore, the activities of HK, PFK, glucose-6-phosphate dehydrogenase $(\mathrm{G} 6 \mathrm{PDH})$, and lactate dehydrogenase (LDH) in breast carcinoma tissues are stimulated by either or both the female sex hormones, circulating estradiol and prolactin (Ramalingam et al., 1994). Moreover, the activities of PFK and $\mathrm{LDH}$ are stimulated by the heat shock protein 70 (Wang et al., 2012).

Thus, the relationship between the calculated periods of the oscillations and $\alpha$ was obtained as follows: $V_{\max }$ was set to $0.65 \mathrm{mM} \mathrm{s}^{-1}$ according to the literature (Marín-Hernández et al., 2014 and Shaw and Amos, 1973); $\alpha$ was changed from 0.05 to 1 , and each $k_{\mathrm{i}}$ was calculated from $\alpha$; next, the $V_{\max }, \alpha$, and $k_{\mathrm{i}}$ values 

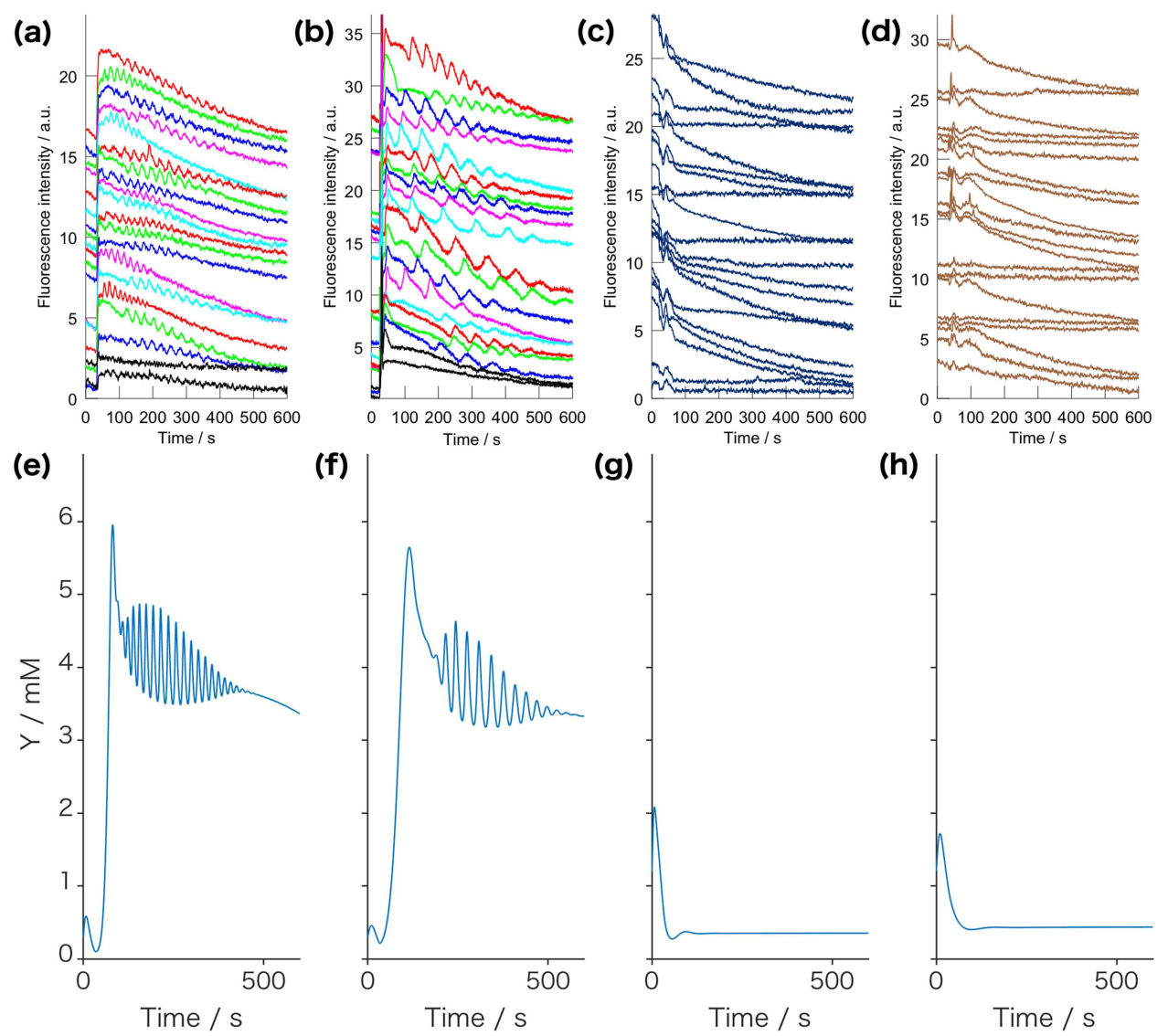

FIG. 2. Typical time series of experimental results with NADH fluorescence [(a)-(d): Reprinted with permission from Amemiya et al., Chaos 27, 104602 (2017). Copyright 2017 American Institute of Physics] and simulation results in an intermediary metabolite $Y[(e)-(h)]$. The pre-incubating conditions are as follows: (a) and (e) (Glc-, FBS+); (b) and (f) (Glc-, FBS-); (c) and (g) (Glc+, FBS+); and (d) and (h) (Glc+, FBS-). The parameter values for the simulation were as follows: rate constants $\left[k_{1}\left(\mathrm{mM}^{-(m+1)} \mathrm{s}^{-1}\right)\right.$, $\left.k_{2}\left(\mathrm{mM}^{-1} \mathrm{~s}^{-1}\right), k_{3}\left(\mathrm{~s}^{-1}\right), k_{4}\left(\mathrm{~s}^{-1}\right)\right]$ under FBS+ and FBS - conditions were $(1.0,0.08,0.1,0.2)$ and $(0.6,0.04,0.08,0.15)$, respectively; parameters $V_{\max }\left(\mathrm{mM} \mathrm{s}^{-1}\right)$ under $\mathrm{Glc}-$ and $\mathrm{Glc}+$ conditions were 0.65 and 0.029 , respectively. The initial concentrations of all intermediates $G(\mathrm{mM}), X(\mathrm{mM}), Y(\mathrm{mM})$, and $A_{3}(\mathrm{mM})$ were 1.2 and 0.3 under $\mathrm{Glc}+$ and $\mathrm{Glc}$ - conditions, respectively, and those in the extracellular solution $G_{\mathrm{ex}}(\mathrm{mM})$ and $Y_{\mathrm{ex}}(\mathrm{mM})$ were set to zero. The values of the other parameters under all the conditions were as follows: $G_{\mathrm{i}}=20 \mathrm{mM}(30 \mathrm{~s} \leq t \leq 32 \mathrm{~s}), A_{0}=3 \mathrm{mM}, K_{\text {eq }}=1, K_{\text {out }}=10 \mathrm{mM}, K_{\text {in }}=12 \mathrm{mM}, K_{1}=1 \mathrm{mM}^{m}, K_{2}=1 \mathrm{mM}^{m}, K_{3}=1 \mathrm{mM}, K_{4}=1 \mathrm{mM}$, $K_{5}=20 \mathrm{mM}^{n}, K_{6}=20 \mathrm{mM}, K_{7}=20 \mathrm{mM}, \kappa=0.01 \mathrm{~s}^{-1}, \varphi=0.1$, and $m=n=4$.

were uniformly randomized from $90 \%$ to $110 \%$; the initial concentrations of $G, X$, and $Y$ were generated from a Gaussian distribution with mean 0.3 and standard deviation 0.053 according to the literature (Nascimento et al., 2016); and the values of $\alpha$ were plotted against

TABLE I. The minimum and maximum values of $k_{1}-k_{4}$, and $a_{i}$ and $b_{i}$ values in Eq. (8).

\begin{tabular}{lccll}
\hline \hline & Minimum value & Maximum value & \multicolumn{1}{c}{$a_{\mathrm{i}}$} & \multicolumn{1}{c}{$b_{\mathrm{i}}$} \\
\hline$k_{1}$ & 0.55 & 1.5 & 1.0 & 0.50 \\
$k_{2}$ & 0.025 & 0.50 & 0.50 & 0 \\
$k_{3}$ & 0.013 & 0.10 & 0.092 & 0.0082 \\
$k_{4}$ & 0.055 & 0.20 & 0.15 & 0.047 \\
\hline \hline
\end{tabular}

periods obtained from the model simulations as shown in Fig. 3 (orange dots; $N=3000$ ). This relationship between the periods $(T)$ and $\alpha$ was fitted to

$$
\alpha=c T^{d},
$$

which is shown by green lines in scatter plots in Fig. 3 with $c=12.7$ and $d=-1.06$.

By using this fitted curve for the relationship between the periods of the oscillations and $\alpha$, distributions of $\alpha$ were calculated from the distributions of the periods obtained experimentally before (Amemiya et al., 2017). Figures 3(a) and 3(b) show the results for glucose-starving (Glc-, FBS+) and both glucose- and serumstarving (Glc-, FBS-) conditions, respectively. The marginal histograms on the scatter plots represent experimental values of the periods, and the histograms of $\alpha$ were obtained from the histograms of the experimental values of the periods by using the fitted curve of 

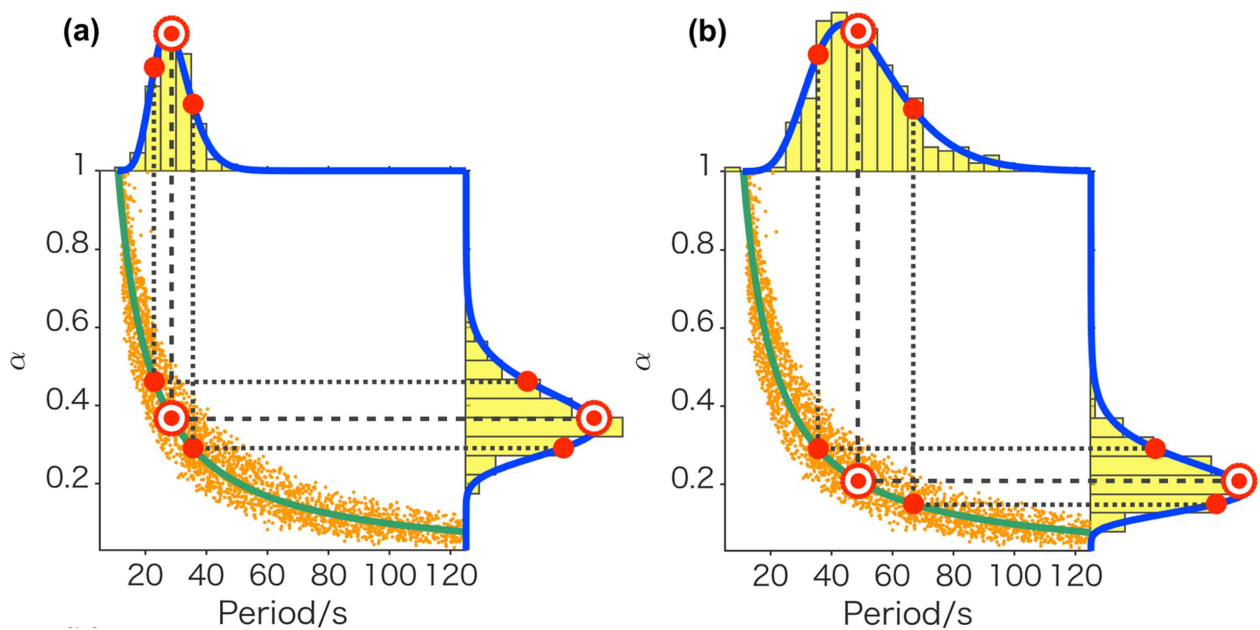

FIG. 3. Relationships between periods and $\alpha . \alpha$ was plotted against periods obtained from the model simulations [orange dots in both panels (a) and (b): $N=3000$ ], which were fit to $\alpha=c T^{d}$ (green lines in scatter plots). The marginal histograms on the scatter plots represent the experimental values of periods: (a) (Glc-, FBS+) and (b) (Glc-, FBS-) conditions [Reprinted with permission from Amemiya et al., Chaos 27, 104602 (2017). Copyright 2017 American Institute of Physics]. The values of $\alpha$ were calculated from the experimental values of periods by the fitted green lines. All histograms were fitted to log-normal distributions (blue lines in the histograms). Red dots with circle: $\exp (\mu)$ of period and alpha; red dots without circle: $\exp (\mu \pm \sigma)$ of period and alpha. The values $(\mu, \sigma)$ in the periods of (Glc-, FBS +$)$ and $(\mathrm{Glc}-$, FBS-) conditions were $(3.5,0.22)$ and $(3.9,0.32)$, respectively. The values $(\mu, \sigma)$ in $\alpha$ of $(\mathrm{Glc}-$, FBS +$)$ and $(\mathrm{Glc}-$, FBS -$)$ conditions were $(-1.0,0.23)$ and $(-1.6,0.33)$, respectively. $\exp (\mu)$ and $\exp (\mu \pm \sigma)$ of period and alpha were correlated by additional gray dashed and dotted lines, respectively. The values of the parameters were the same as those in Fig. 2 except for $V_{\max }, k_{\mathrm{i}}(i=1-4)$, and the initial concentrations of $G, X$, and $Y$; the values of $V_{\max }, k_{i}, \alpha$, and the initial concentrations were randomized (see the text for details).

Eq. (9). These histograms were fitted to log-normal distributions as shown by blue lines in Figs. 3(a) and 3(b). It is noted that all distributions of periods and $\alpha$ were log-normal distribution according to the Anderson-Darling test $(p<0.05)$; the previous study (Amemiya et al., 2017) fitted the period distribution to normal distribution function under the (Glc-, FBS+) condition, but log-normal distribution is more appropriate for it.

We observe that the distribution of the values of $\alpha$, i.e., the rate constants, is large. For instance, $\alpha=0.29-0.46\left(=e^{-1.0 \pm 0.23}\right)$ under (Glc-, FBS+), indicating that the metabolic heterogeneity in $\mathrm{HeLa}$ cells is significant. Moreover, (Glc-, FBS-) is found to enhance the metabolic heterogeneity in HeLa cells as shown in Fig. 3(b).

\section{Simulation of the ratio of oscillating cells with time}

The previous experiments showed that not all HeLa cells exhibited glycolytic oscillations; the maximum oscillatory ratios were about 0.4 and 0.6 for (Glc-, FBS+) and (Glc-, FBS-) conditions, respectively (Amemiya et al., 2017). In addition, the starting time and duration of the oscillations were also different among the oscillated cells. In order to simulate the oscillatory characteristics in HeLa cells, we first investigated the relationship between the oscillatory ratio and the maximum velocity $\left(V_{\max }\right.$ ) of glucose uptake through GLUT, since our previous experiments and the present simulations (Fig. 2) indicate that HeLa cells exhibit no oscillations when the value of $V_{\max }$ is small due to no-starvation of glucose.

Figure 4 shows the relationship between the oscillatory ratio and $V_{\max }$ under the conditions (Glc-, FBS+) and (Glc-, FBS-), respectively. The oscillatory ratio as a function of $V_{\max }$ was calculated as follows.

According to Fig. 3, the location parameters $\mu$ of $\alpha$ (i.e., mean value of natural logarithm of $\alpha$ ) was -1.0 and -1.6 for (Glc-, FBS + ) and (Glc-, FBS-), respectively; the scale parameters $\sigma$ of $\alpha$ (i.e., standard deviation of the natural logarithm of $\alpha$ ) were 0.23 and 0.33 for (Glc-, FBS+) and (Glc-, FBS-), respectively. To demonstrate the variability in the activity of a cell and the variabilities in the enzyme activity in the cell, both $\alpha$ and each of $k_{1}-k_{4}$ were randomized, independently. However, since $k_{1}-k_{4}$ are randomized twice through this randomizing process (i.e., random numbers for $\alpha$ and $\left.k_{\mathrm{i}}\right), \sigma / \sqrt{2}$ was used for each step, which gives a final scale parameter $\sigma$ because of the reproducibility of the log-normal distribution assuming that the scale parameters of $\alpha$ and $k_{\mathrm{i}}$ are equal. Additionally, the initial concentrations of $G, X$, and $Y$ were generated from the Gaussian distribution with the mean value of 0.3 and the standard deviation of 0.053 . For each $V_{\max }$ (100 points that are evenly spaced between 0.029 and 0.65$)$, the ratio of oscillatory parameter sets was calculated $(N=3000)$. Oscillations are determined as a sequence of peaks whose amplitudes are larger than 1 and repeated more than 3 times.

Figure 4 clearly shows that the oscillatory ratio decreases with the decrease in the value of $V_{\max }$. In addition, the oscillatory ratio is larger under the condition of both glucose- and serum-starvation (Glc-, FBS-) than that of only glucose-starvation (Glc-, FBS+), which agrees with the previous experiments as well as with the simulation as shown below. Thus, we can say that starvation of glucose induces high uptake of glucose (Shaw and Amos, 1973), leading to HeLa cells to exhibit glycolytic oscillations with a high probability. 


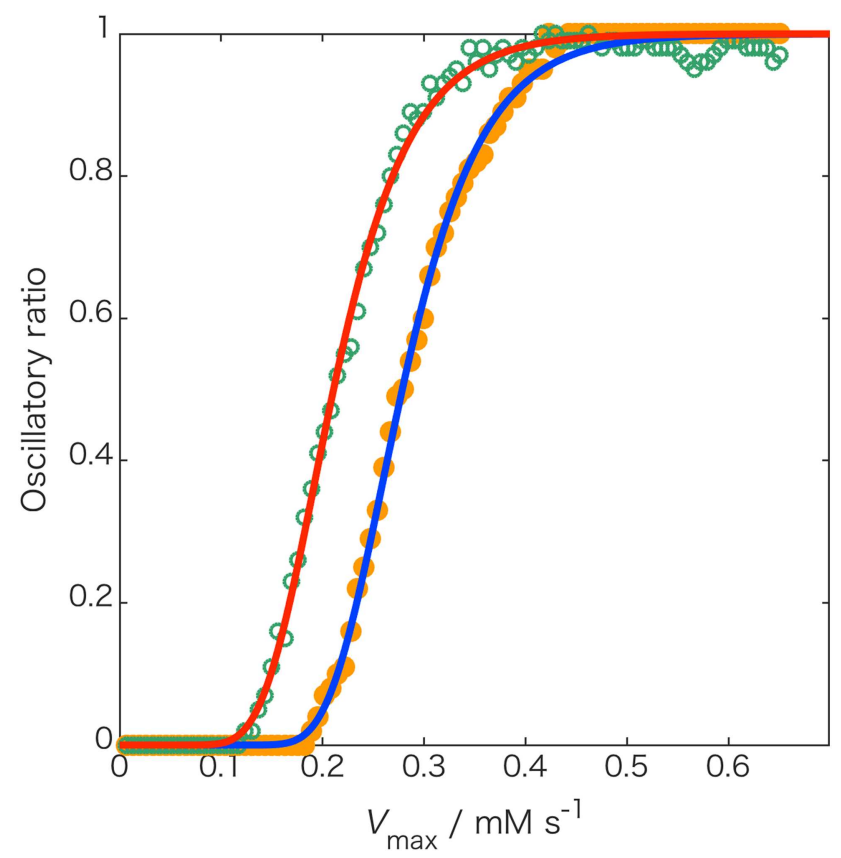

FIG. 4. Dependence of oscillatory ratio on $V_{\max } . V_{\max }$ was fixed to each value, and the numbers of oscillatory cells to those of both non-oscillatory and oscillatory cells (oscillatory ratio) were calculated ( $N=1000$ for each point). Filled orange circles and open green circles were the results under (Glc-, FBS+) and (Glc-, FBS-), respectively. Blue and red solid lines are fitted lines under (Glc-, FBS+) and ( $\mathrm{Gl} c-, \mathrm{FBS}-$ ), respectively. The values of the parameters were the same as those in Fig. 2 except for $V_{\max }, k_{i}(i=1-4)$, and the initial concentrations of $G, X$, and $Y$; the values of $V_{\max }, k_{i}, \alpha$, and the initial concentrations were randomized (see the text for details).

Afterwards, we simulated the experimental results of the oscillatory ratio as a function of time by considering the effect of $V_{\max }$ on the oscillatory ratio. Figure 5 shows that the simulated results of the oscillatory ratio as a function of time reproduce the experimental results very well. The numerical simulations were carried out as follows: the randomizing methods for $G, X, Y, \alpha$, and $k_{\mathrm{i}}$, as well as oscillation detections, were the same as in Fig. 3. $V_{\max }$ was generated from the log-normal distribution with $\mu=-1.4$ and $\sigma=0.16$.

It is noted that smaller mean values of $V_{\max }$ were used for the simulation than that used in Fig. $3\left(0.65 \mathrm{mM} \mathrm{s}^{-1}\right)$. This takes into account the fact that many non-oscillatory cells existed in previous experiments, as shown in panels (a) and (b) in Fig. 5, indicating that many cells might have smaller uptake velocity $\left(V_{\max }\right)$ of glucose than that used for the simulation as shown in Fig. 3. The present model always produces oscillations under the simulated conditions as shown in Fig. 3. Thus, it is reasonable to use a smaller mean value of $V_{\max }$ for the simulation of the oscillatory ratio vs. time.

Figures 5(e) and 5(f) show the period distributions obtained from time series of the oscillations for the oscillatory cells [Figs. 5(c) and $5(\mathrm{~d})]$. The geometric means of the periods for the glucosestarving (Glc-, FBS+) and both glucose- and serum-starving (a)
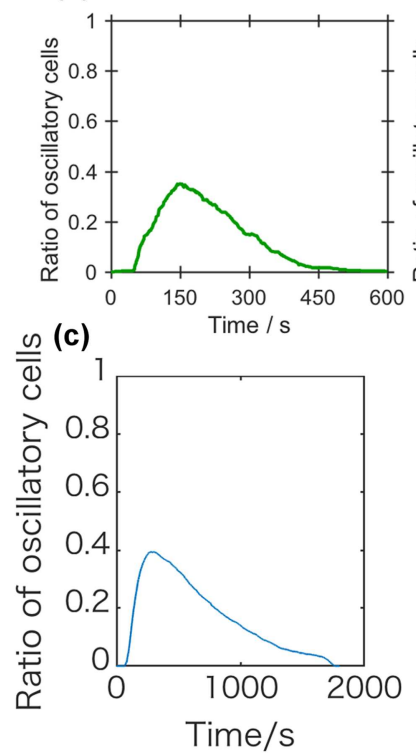

(e)

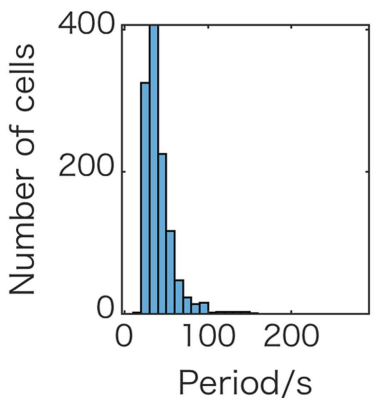

(b)
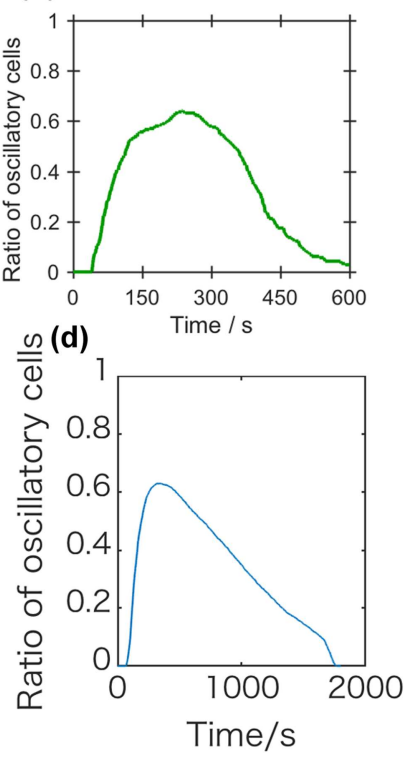

(f)

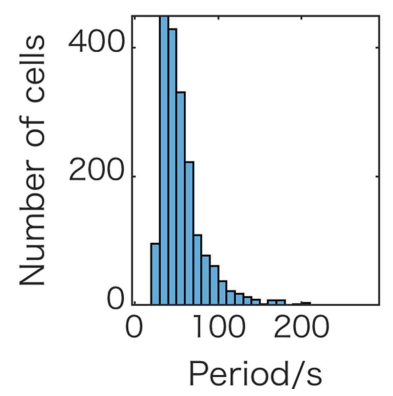

FIG. 5. Simulation of the ratios of oscillatory cells. Experimental results of ratios of oscillatory cells [(a) and (b): $N=255$ and 212 under the (Glc-, FBS+) and (Glc-, FBS-) conditions, respectively; Reprinted with permission from Amemiya et al., Chaos 27, 104602 (2017). Copyright 2017 American Institute of Physics]. Simulations of ratios of oscillatory cells [(c) and (d): $N=3000]$, and their distributions of the periods $[(\mathrm{e})$ and $(\mathrm{f})]$. The values of the parameters were the same as those in Fig. 4 except for $V_{\max } ; V_{\max }$ was generated from the log-normal distribution with $\mu=-1.4$ and $\sigma=0.16$, and the other values of $\alpha, k_{\mathrm{i}}$, and the initial concentrations were randomized as in Fig. 4 (see the text for details).

(Glc-, FBS-) conditions were 38 and $52 \mathrm{~s}$, respectively, and these values agree with the experimental results [Figs. 3(a) and 3(b)].

\section{DISCUSSION}

We have developed a simple mathematical model for glycolytic oscillations in HeLa cervical cancer cells. The present study reveals that the mechanism of glycolytic oscillations in HeLa cells can be explained by the framework of that for yeast cells (Goldbeter, 1996; Sel'kov, 1968; and Wolf et al., 2000). The present model can simulate the previous experimental results of glycolytic oscillations in individual HeLa cells quantitatively. The effect of glucose-starvation 
in HeLa cells is reported to increase the uptake velocity of glucose through glucose transporter GLUT (Shaw and Amos, 1973) and can be modeled by increasing the value of the maximum velocity $\left(V_{\max }\right)$ of GLUT in the present model. Contrarily, HeLa cells with noglucose starvation exhibited no oscillations, which can be modeled by decreasing the value of $V_{\max }$. Glucose starvation is also indispensable for yeast cells to exhibit glycolytic oscillations; however, the effect of glucose starvation seems to be controversial between HeLa cells (Shaw and Amos, 1973) and yeast cells (Alberts et al., 2007). In the case of yeast, $S$. cerevisiae, decrease in the hexose uptake kinetics is reported as a result of carbon-, nitrogen-, or both carbon- and nitrogen-starvation (Alberts et al., 2007). For the moment, we cannot explain the controversial effects of glucose starvation on HeLa and yeast cells.

Serum is indispensable for mammalian cells to grow; however, serum-starvation is frequently used to adjust physiological states in mammalian cells (Pirkmajer and Chibalin, 2011). In relation to the present study, the activity of PFK, the core regulatory enzyme of glycolytic oscillations, is reported to increase by the serum addition to serum-starved cultures of human fibroblasts (Bruni et al., 1983). Thus, the activity of PFK, as well as the other glycolytic enzymes in HeLa cells, might be decreased during serum-starving conditions. The present study has modeled the effect of serum starvation as the decrease in the values of all four rate constants of the reaction steps and succeeded in reproducing the prolongation of the periods of the oscillations (Fig. 2).

Oscillatory cells exhibited intercellular heterogeneity in the periods of the oscillations, and the period distributions have been attributed to the distribution of the values of all (four) rate constants. It is to be noted that significantly different concentrations of all four key enzymes in different cells may occur, since there is strong competition for amino acids in cancer cells in the processes of enzyme synthesis. This possibility has been taken into account in the rate constants; the two rate constants, $k_{1}$ and $k_{2}$, for PFK and PK reactions, respectively, include the concentrations of the enzymes in such a way that $k_{1}=\left(k_{1}{ }^{\prime} / K_{1} / K_{3}+k_{2}{ }^{\prime} / K_{1} / K_{4}\right) E_{0}$ and $k_{2}=\left(k_{3}{ }^{\prime} / K_{6}+k_{4}{ }^{\prime} / K_{7}\right) E_{0}{ }^{\prime}$, respectively, which can be found in the Appendix. On the other hand, the rest of the two rate constants, $k_{3}$ and $k_{4}$, also include the concentrations of enzymes since they are assumed to be the product of the specific activity and the concentrations of enzymes.

In order to investigate how the period distributions emerge, the values of the four rate constants as well as the maximum uptake velocity $\left(V_{\max }\right)$ of glucose were randomized in the model, and the periods of the oscillations upon addition of glucose were examined. In this calculation, the parameter $\alpha$ was introduced as presented by Eq. (8) in order to present intercellular heterogeneity in enzymatic activity, and each of the four values of the rate constants was uniformly randomized from $90 \%$ to $110 \%$. Equation (8) assumes that these rate constants can be different among HeLa cells; however, they are not totally random but have a tendency to be large or small in a linear relation. Namely, we did not assume that the activity of one of the enzymes was very high, whereas the activity of one of the other three enzymes was very low in a cell.

This assumption for intercellular heterogeneity in enzymatic activity gave the relationship between the periods of the oscillations and the parameter $\alpha$ as shown in Fig. 3, and then the experimentally obtained period distributions were transformed to the distributions of the values of the four rate constants. By using these distributions, the oscillatory ratio $(N=3000)$ as a function of time was reproduced quantitatively as shown in Fig. 5.

Heterogeneity in glycolytic oscillations has been reported in yeast cells immobilized on a coverslip, agarose pads, or in a microfluidic chamber (Weber et al., 2012; Dodd and Kralj, 2017; and Gustavsson et al., 2012). However, the degree of the heterogeneity is different depending on the strains and experimental conditions. S. cerevisiae (strain $\mathrm{X} 2180$ ) exhibited oscillations homogeneously in terms of their periods of $55 \pm 5.6 \mathrm{~s}$; however, very heterogeneous in terms of start time, stop time, and duration in a microfluidic chamber (Gustavsson et al., 2012). Saccharomyces carlsbergensis (strain ATCC 9080) exhibited oscillations heterogeneously with different periods (ca. 39-65 s) at a low cell density; however, homogeneously in terms of their periods (ca. 39-42 s) and phases at a high cell density on a coverslip (Weber et al., 2012). S. cerevisiae (strain BY4741) is reported to oscillate very heterogeneous in period (ca. 29-63 s), start time, stop time, duration, and amplitude on agarose pads (Dodd and Kralj, 2017). The reasons for these heterogeneities in yeast glycolytic oscillations are currently unknown; nevertheless, in the first case, S. cerevisiae (strain X2180), homogeneous distributions in the periods of oscillations are explained by small variations $( \pm 2 \%)$ in the maximum velocity $\left(V_{\max }\right)$ of glucose uptake, which is reported to be sufficient to simulate the most $(96.2 \%)$ of the experimental results (Gustavsson et al., 2012).

The present study shows that a strong heterogeneity in the glycolytic oscillations in HeLa cells may arise from large distributions in the values of the rate constants, i.e., enzymatic activity, which are expressed by lognormal distributions as shown in Fig. 3. Different initial values of the metabolites (initial conditions of the variables in the model) were allowed in the present simulations, however, affected the results little.

Cancer cells composing tumor tissues are generally known to be heterogeneous, called intratumor heterogeneity, at gene levels (Gerlinger et al., 2012). This may be the cause of the heterogeneity in the enzymatic activity in HeLa cells, leading to strong heterogeneous glycolytic oscillations.

\section{ACKNOWLEDGEMENTS}

We are grateful to Dr. Showalter for his ongoing leadership in nonlinear science. We thank Professor Kazuyuki Nakamura of MIMS for useful discussion. This study was supported in part by a grant for Cooperative Research Project to T.A. from YNU and KAKENHI grant (No. 17KT0123) to S.N. from the Japan Society for the Promotion of Science (JSPS).

\section{APPENDIX: REACTION MECHANISMS FOR THE PFK AND PK REACTIONS}

The reaction scheme for the PFK reaction is assumed to be the following:

$$
\begin{gathered}
E_{\mathrm{R}} \stackrel{K_{1}}{\leftrightarrow} E+m A_{2}, \\
E_{\mathrm{T}} \stackrel{K_{2}}{\leftrightarrow} E+m A_{3}, \\
\left(E_{R} G\right) \stackrel{K_{3}}{\leftrightarrow} E_{R}+G,
\end{gathered}
$$




$$
\begin{gathered}
\left(E_{R} A_{3}\right) \stackrel{K_{4}}{\leftrightarrow} E_{R}+A_{3}, \\
\left(E_{R} G\right)+A_{3} \stackrel{k_{1}^{\prime}}{\rightarrow} X+A_{2}+E_{R}, \\
\left(E_{R} A_{3}\right)+G \stackrel{k_{2}^{\prime}}{\rightarrow} X+A_{2}+E_{R}, \\
E+E_{R}+E_{T}+\left(E_{R} G\right)+\left(E_{R} A_{3}\right)=E_{0} .
\end{gathered}
$$

Here, reactions (A1) and (A2) represent conformational transitions of the free enzyme PFK $(E)$ to $E_{\mathrm{R}}$ and $E_{\mathrm{T}}$ states by activation and inhibition with $m$-molecules of ADP $\left(A_{2}\right)$ and ATP $\left(A_{3}\right)$, respectively. These dissociation constants are represented by $K_{1}$ and $K_{2}$, respectively. Reactions (A3)-(A6) are the PFK reactions in the form of the activated state $E_{\mathrm{R}}$ with its two substrates $G$ and ATP $\left(A_{3}\right)$, respectively. The dissociation constants for (A3) and (A4) are represented by $K_{3}$ and $K_{4}$, respectively. Here, the present model assumes glucose $(G)$ as one of the substrates for the PFK reaction instead of fructose 6-phosphate in order to keep the model as simple as possible with the limited number of the variables. Reactions (A3) and (A5) are the case where $G$ binds to PFK first, and subsequently, ATP binds to PFK, whereas (A4) and (A6) are the cases where ATP binds to PFK first, and subsequently $G$ binds to PFK. Equation (A7) is the mass balance of the enzyme of PFK, in which $E_{0}$ represents the total concentration of PFK.

In the present study, the reaction rate $v_{1}$ is assumed to be the sum of the production rate of $X$

$$
v_{1}=\frac{\mathrm{d} X}{\mathrm{~d} t}=k_{1}^{\prime}\left(E_{R} G\right) A_{3}+k_{2}^{\prime}\left(E_{R} A_{3}\right) G .
$$

The above rate law resulted in Eq. (6a) in the text by replacing $k_{1}=\left(\frac{k_{1}^{\prime}}{K_{1} K_{3}}+\frac{k_{2}^{\prime}}{K_{1} K_{4}}\right) E_{0}$, and

$$
A_{2}=A_{0}-A_{3} \text {. }
$$

In the same way, the reaction scheme for the $\mathrm{PK}$ reaction is assumed to be the following:

$$
\begin{gathered}
E_{T}^{\prime} \stackrel{K_{5}}{\leftrightarrow} E^{\prime}+n A_{3}, \\
\left(E^{\prime} X\right) \stackrel{K_{6}}{\leftrightarrow} E^{\prime}+X, \\
\left(E^{\prime} A_{2}\right) \stackrel{K_{7}}{\leftrightarrow} E^{\prime}+A_{2}, \\
\left(E^{\prime} X\right)+A_{2} \stackrel{k_{3}^{\prime}}{\rightarrow} Y+A_{3}+E^{\prime}, \\
\left(E^{\prime} A_{2}\right)+X \stackrel{k_{4}^{\prime}}{\rightarrow} Y+A_{3}+E^{\prime}, \\
E^{\prime}+E_{T}^{\prime}+\left(E^{\prime} X\right)+\left(E^{\prime} A_{2}\right)=E_{0}^{\prime},
\end{gathered}
$$

where $E^{\prime}$ represents the free form of the enzyme $\mathrm{PK}, E_{\mathrm{T}}{ }^{\prime}$ is the allosterically inhibited form of PK by $n$-molecules of ATP, and $E_{0}{ }^{\prime}$ is the total concentration of $\mathrm{PK}$. The reaction rate $v_{2}$ is the production rate of $Y$ given by

$$
v_{2}=\frac{\mathrm{d} Y}{\mathrm{~d} t}=k_{3}^{\prime}\left(E^{\prime} X\right) A_{2}+k_{4}^{\prime}\left(E^{\prime} A_{2}\right) X
$$

The above rate law resulted in Eq. (6b) in the text by replacing $k_{2}=\left(\frac{k_{3}^{\prime}}{K_{6}}+\frac{k_{4}^{\prime}}{K_{7}}\right) E_{0}^{\prime}$.

\section{REFERENCES}

Alberts, E., Larsson, C., Andlid, T., Walsh, M. C., and Gustafsson, L., "Effect of nutrient starvation on the cellular composition and metabolic capacity of Saccharomyces cerevisiae," Appl. Environ. Microbiol. 73(15), 4839-4848 (2007).

Amemiya, T., Obase, K., Hiramatsu, N., Itoh, K., Shibata, K., Takinoue, M., Yamamoto, T., and Yamaguchi, T., "Collective and individual glycolytic oscillations in yeast cells encapsulated in alginate microparticles," Chaos 25, 064606 (2015).

Amemiya, T., Shibata, K., Itoh, Y., Itoh, K., Watanabe, M., and Yamaguchi, T., "Primordial oscillations in life: Direct observation of glycolytic oscillations in individual HeLa cervical cancer cells," Chaos 27, 104602 (2017).

Bruni, P., Farnararo, M., Vasta, V., and D'Alessandro, A., "Increase of the glycolytic rate in human resting fibroblasts following serum stimulation," FEBS Lett. 159, 39-42 (1983).

Chance, B. and Hess, B., "Metabolic control mechanisms: IV. The effect of glucose upon the steady state of respiratory enzymes in the ascites cell," J. Biol. Chem. 234, 2421-2427 (1959).

Chandra, F. A., Buzi, G., and Doyle, J. C., "Glycolytic oscillations and limits on robust efficiency," Science 333, 187-192 (2011).

Crabtree, H. G., "Observations on the carbohydrate metabolism of tumors," Biochem. J. 23, 536-545 (1929).

Danø, S., Sørensen, P., and Hynne, F., "Sustained oscillations in living cells," Nature 402, 320-322 (1999).

De Monte, S., d'Ovidio, F., Danø, S., and Sørensen, P., “Dynamical quorum sensing: Population density encoded in cellular dynamics," Proc. Natl. Acad. Sci. U.S.A. 20, 18377-18381 (2007).

Dodd, B. J. T. and Kralj, J. M., "Live cell imaging reveals $\mathrm{pH}$ oscillations in Saccharomyces cerevisiae during metabolic transitions," Sci. Rep. 7, 13922 (2017).

Diaz-Ruiz, R., Rigoulet, M., and Devin, A., "The Warburg and Crabtree effects: On the origin of cancer cell energy metabolism and of yeast glucose repression," Biochim. Biophys. Acta 1807, 568-576 (2011).

du Preez, F. B., van Niekerk, D. D., and Snoep, J. L., "From steady-state to synchronized yeast glycolytic oscillations II: Model validation," FEBS J. 279, 2823-2836 (2012).

Gerlinger, M. et al., "Intratumor heterogeneity and branched evolution revealed by multiregion sequencing," N. Engl. J. Med. 366, 883-892 (2012).

Goldbeter, A., Biochemical Oscillations and Cellular Rhythms (Cambridge University Press, 1996).

Goldbeter, A. and Lefever, R., "Dissipative structure for an allosteric model," Biophys. J. 12, 1302-1315 (1972).

Gustavsson, A.-K., van Niekerk, D. D., Adiels, C. B., du Preez, F. B., Goksör, M., and Snoep, J. L., "Sustained glycolytic oscillations in individual isolated yeast cells," FEBS J. 279, 2837-2847 (2012).

Hald, B. O. and Sørensen, P. G., "Modeling diauxic glycolytic oscillations in yeast," Biophys. J. 99, 3191-3199 (2010).

Hamanaka, R. B. and Chandel, N. S., "Targeting glucose metabolism for cancer therapy," J. Exp. Med. 209, 211-215 (2012).

Heiden, M. G. V., Cantley, L. C., and Thompson, C. B., "Understanding the Warburg effect: The metabolic requirements of cell proliferation," Science 324, 1029-1033 (2009).

Ibsen, K. H. and Schiller, K. W., "Oscillations of nucleotides and glycolytic intermediates in aerobic suspensions of Ehrlich ascites tumor cells," Biochim. Biophys. Acta 131, 405-407 (1967).

Khazaei, T., McGuigan, A., and Mahadevan, R., "Ensemble modeling of cancer metabolism," Front. Physiol. 3, 135 (2012).

Marín-Hernández, A., Gallardo-Pérez, J. C., Rodríguez-Enríquez, S., Encalada, R., Moreno-Sánchez, R., and Saavedra, E., "Modeling cancer glycolysis," Biochem. Biophys. Acta 1807, 755-767 (2011).

Marín.-Hernández, A., López-Ramirez, S. Y., Mazo.-Monsalvo, I. D., GallardoPerez, J. C., Rodríguez-Enriquez, S., Moreno-Sanchez, R., and Saavera, E., "Modeling cancer glycolysis under hypoglycemia, and the role played by the differential expression of glycolytic isoforms," FEBS J. 281, 3325-3345 (2014).

Martin, R. R., Montero, S., Silva, E., Bizzarri, M., Cocho, G., Mansilla, R., and NietoVillar, J. M., "Phase transitions in tumor growth: $\mathrm{V}$ what can be expected from cancer glycolytic oscillations?,” Phys. A 486, 762-771 (2017). 
Nascimento, R. A., Özel, R. E., Mak, W. H., Mulato, M., Singaram, B., and Pourmand, N., "Single cell glucose nanosensor verifies elevated glucose levels in individual cancer cells," Nano Lett. 16, 1194-1200 (2016).

Olsen, L. F., Andersen, A. Z., Lunding, A., Brasen, J. C., and Poulsen, A. K., "Regulation of glycolytic oscillations by mitochondrial and plasma membrane $\mathrm{H}^{+}$-ATPases," Biophys. J. 96, 3850-3861 (2009).

Pirkmajer, S. and Chibalin, A. V., "Serum starvation: Caveat emptor," Am. J. Physiol. Cell Physiol. 301, C272-C279 (2011).

Ramalingam, V., Krishnamoorthy, G., and Govindarajulu, P., "Enzymes of carbohydrate metabolism in human breast carcinoma: Relationship with serum hormones," Neoplasma 41, 49-54 (1994).

Richard, P., Bakker, B. M., Teusink, B., van Dam, K., and Westerhoff, H. V., "Acetaldehyde mediates the synchronization of sustained glycolytic oscillations in populations of yeast cells," Eur. J. Biochem. 235, 238-241 (1996).

Schütze, J. and Wolf, J., "Spatio-temporal dynamics of glycolysis in cell layers. A mathematical model," Biosystems 99, 104-108 (2010).

Sel'kov, E. E., "Self-oscillations in glycolysis. 1. A simple kinetic model," Eur. J. Biochem. 4, 79-86 (1968).

Shaw, S. N. and Amos, H., "Insulin stimulation of glucose entry in chick fibroblasts and HeLa cells,” Biochem. Biophys. Res. Commun. 53, 357-365 (1973).

Shibata, K., Amemiya, T., Kawakita, Y., Obase, K., Itoh, K., Takinoue, M., Nakata, S., and Yamaguchi, T., "Promotion and inhibition of synchronous glycolytic oscillations in yeast by chitosan," FEBS J. 285, 2679-2690 (2018).

Shinomoto, S. and Kuramoto, Y., "Phase transition in active rotator systems," Prog. Theor. Phys. 75, 1105-1110 (1986).
Taylor, A. F., Tinsley, M. R., Wang, F., Huang, Z., and Showalter, K., "Dynamical quorum sensing and synchronization in large populations of chemical oscillators," Science 323, 614-617 (2009).

Termonia, Y. and Ross, J., "Oscillations and control features in glycolysis: Numerical analysis of a comprehensive model," Proc. Natl. Acad. Sci. U.S.A. 78, 2952-2956 (1981).

Wang, L., Schumann, U., Liu, Y., Prokopchuk, O., and Steinacker, J. M., "Heat shock protein 70 (Hsp 70) inhibits oxidative phosphorylation and compensates ATP balance through enhanced glycolytic activity," J. Appl. Physiol. 113, 1669-1676 (2012).

Warburg, O., "On the origin of cancer cells,” Science 123, 309-314 (1956).

Weber, A., Prokazov, Y., Zuschratter, W., and Hauser, M. J. B., "Desynchronisation of glycolytic oscillations in yeast cell populations," PLoS One 7, e43276 (2012).

Wolf, J. and Heinrich, R., "Effect of cellular interaction on glycolytic oscillations in yeast: A theoretical investigation," Biochem. J. 345, 321-334 (2000).

Wolf, J., Passarge, J., Somsen, O. J. G., Snoep, J. L., Heinrich, R., and Westerhoff, H. V., "Transduction of intracellular and intercellular dynamics in yeast glycolytic oscillations," Biophys. J. 78, 1145-1153 (2000).

Wu, R. and Racker, E., "Regulatory mechanisms in carbohydrate metabolism. IV. Pasteur effect and Crabtree effect in ascites tumor cells," J. Biol. Chem. 234, 1036-1041 (1959).

Zheng, N., Wang, K., He, J., Qiu, Y., Xie, G., Su, M., Jia, W., and Li, H., "Effects of ADMA on gene expression and metabolism in serum-starved LoVo cells," Sci. Rep. 6, 25892 (2016). 